\title{
LOCALIZATION ANALYSIS OF DAMAGE FOR ONE-DIMENSIONAL PERIDYNAMIC MODEL
}

\author{
Karel Mikešs ${ }^{a, *}$, Milan JiráseK ${ }^{a}$, Jan Zeman ${ }^{a}$, Ondřej Rokoš ${ }^{b}$, \\ Ron H. J. PEerLings ${ }^{b}$ \\ ${ }^{a}$ Czech Technical University in Prague, Faculty of Civil Engineering, Department of Mechanics, Thákurova 7 , \\ 16629 Prague, Czech Republic \\ ${ }^{b}$ Eindhoven University of Technology, Department of Mechanical Engineering, PO Box 513, 5600 MB \\ Eindhoven, The Netherlands \\ * corresponding author: Karel.Mikes@cvut.cz
}

Abstract. Peridynamics is a recently developed extension of continuum mechanics, which replaces the traditional concept of stress by force interactions between material points at a finite distance. The peridynamic continuum is thus intrinsically nonlocal. In this contribution, a bond-based peridynamic model with elastic-brittle interactions is considered and the critical strain is defined for each bond as a function of its length. Various forms of length functions are employed to achieve a variety of macroscopic responses. A detailed study of three different localization mechanisms is performed for a one-dimensional periodic unit cell. Furthermore, a convergence study of the adopted finite element discretization of the peridynamic model is provided and an effective event-driven numerical algorithm is described.

KEYWORDs: Damage, localization, nonlocal continuum, peridynamics.

\section{BOND-BASED PERIDYNAMICS}

For natural as well as man-made materials, the resulting macroscopic response is dictated by the finescale structure of the material. Individual building units (such as atoms on the nanoscale, inclusions and matrix on the microscale, or bricks on the mesoscale) have a certain characteristic size and their interaction takes place at finite distance. This can be taken into account by particle/lattice models, or by various enriched continuum theories. In this work, we consider the bond-based version of the peridynamic model and study the effect of the specific choice of the critical strain function on the evolution and localization of the macroscopic damage.

Peridynamics was introduced in 2000 by S. A. Silling as a reformulation of the classical theory of elasticity [1]. In peridynamics, the partial differential equations of the classical continuum theory are replaced by integral equations. Since differentiation of the displacement field is not needed, peridynamics is especially convenient for capturing discontinuities such as cracks without the complications of mathematical singularities.

In a peridynamic model, the material is represented by infinitely many infinitely small particles that can interact at a finite distance. Usually, interaction between two points is considered only if their distance does not exceed the so-called horizon $h$, see Figure 1.

The acceleration $\ddot{\mathbf{u}}$ of a particle located at a spatial position $\mathbf{x}$ in the reference configuration at time $t$ can

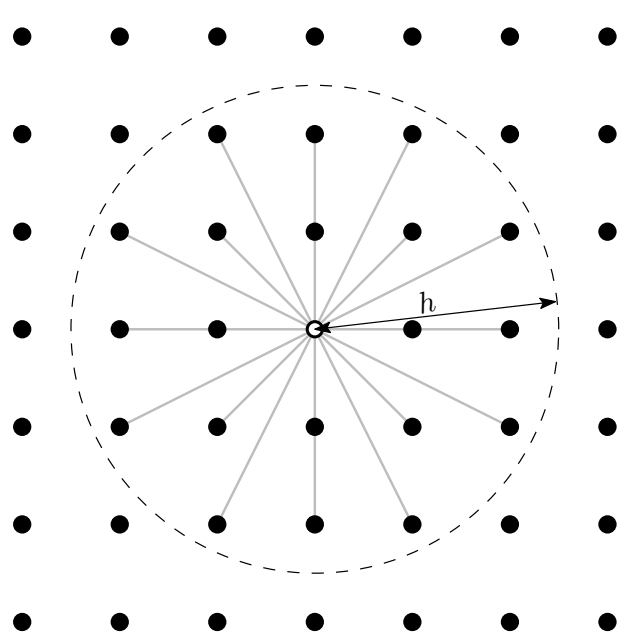

Figure 1. Two-dimensional representation of a discretized peridynamic model: regular grid of particles (black dots) and one selected particle (hollow circle) with interactions (grey lines) within horizon $h$ (dashed line).

be found from the peridynamic equation of motion

$$
\begin{array}{r}
\rho \ddot{\mathbf{u}}(\mathbf{x}, t)=\int_{\mathcal{H}_{x}} \mathbf{f}\left(\mathbf{u}\left(\mathbf{x}^{\prime}, t\right)-\mathbf{u}(\mathbf{x}, t), \mathbf{x}^{\prime}-\mathbf{x}\right) \mathrm{d} V_{\mathbf{x}^{\prime}} \\
+\mathbf{b}(\mathbf{x}, t) \\
\rho \ddot{\mathbf{u}}(\mathbf{x}, t)=\int_{\mathcal{H}_{x}} \mathbf{f}\left(\mathbf{u}\left(\mathbf{x}^{\prime}, t\right)-\mathbf{u}(\mathbf{x}, t), \mathbf{x}^{\prime}-\mathbf{x}\right) \mathrm{d} V_{\mathbf{x}^{\prime}}+\mathbf{b}(\mathbf{x}, t)
\end{array}
$$

where $\mathcal{H}_{x}$ is the set of points that interact with the particle at $\mathbf{x}, \mathbf{u}$ is the displacement vector field, $\mathbf{b}$ 

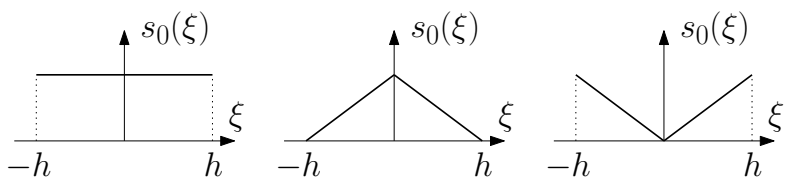

Figure 2. Various functions describing the dependence of failure strain on the initial bond length: constant (left), decreasing (middle), and increasing (right).

is the prescribed body force density field, $\rho$ is the mass density in the reference configuration, and $\mathbf{f}$ is the so-called pairwise force function. The value of f is the force vector (per unit volume squared) that a particle located at the position $\mathbf{x}^{\prime}$ exerts on the particle located at $\mathbf{x}$. The pairwise force function can be written in the form

$$
\mathbf{f}(\boldsymbol{\eta}, \boldsymbol{\xi})=\frac{\boldsymbol{\xi}+\boldsymbol{\eta}}{|\boldsymbol{\xi}+\boldsymbol{\eta}|} f(|\boldsymbol{\xi}+\boldsymbol{\eta}|, \boldsymbol{\xi})
$$

where

$$
\xi=\mathrm{x}^{\prime}-\mathrm{x}
$$

is the relative position of the interacting particles in the reference configuration,

$$
\boldsymbol{\eta}=\mathbf{u}\left(\mathbf{x}^{\prime}, t\right)-\mathbf{u}(\mathbf{x}, t)
$$

is their relative displacement, and $f$ is the scalar bond force governed by a constitutive relation.

In this work, bonds are considered as perfectly elastic-brittle, in line with the concept of microelastic brittle material, adopted from [2]. The corresponding bond force is defined as

$$
f(|\boldsymbol{\xi}+\boldsymbol{\eta}|, \boldsymbol{\xi})=c s(|\boldsymbol{\xi}+\boldsymbol{\eta}|,|\boldsymbol{\xi}|) \mu(t, \boldsymbol{\xi})
$$

where $c$ is a spring constant called the bond elastic micromodulus,

$$
s(|\boldsymbol{\xi}+\boldsymbol{\eta}|,|\boldsymbol{\xi}|)=\frac{|\boldsymbol{\xi}+\boldsymbol{\eta}|-|\boldsymbol{\xi}|}{|\boldsymbol{\xi}|}
$$

is the bond stretch and

$$
\mu(t, \boldsymbol{\xi})= \begin{cases}1 & \text { if } s\left(t^{\prime}, \boldsymbol{\xi}\right)<s_{0} \text { for all } 0 \leq t^{\prime} \leq t \\ 0 & \text { otherwise }\end{cases}
$$

is a history-dependent damage function that jumps from 1 to 0 when the bond breaks, which is assumed to happen when its stretch attains the critical level, $s_{0}$.

In this work, a fixed value of the elastic micromodulus $c$ is considered for all bonds, whereas the critical stretch for bond failure, $s_{0}$, is evaluated for each link as a function of its length. Three different forms of length functions, namely constant, linear decreasing, and linear increasing, are employed, as illustrated in Figure 2.

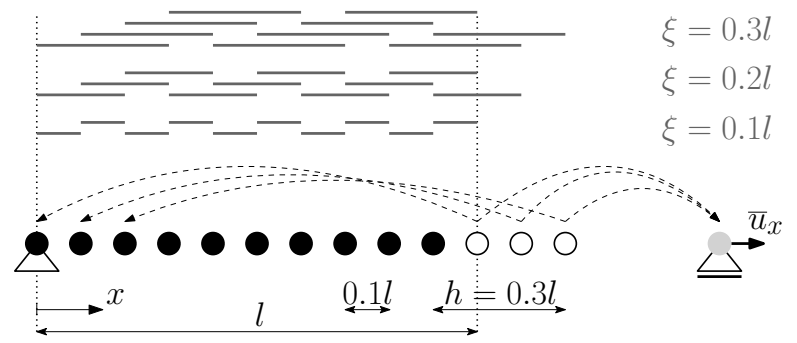

FiguRE 3. Geometry of an illustrative example of the one-dimensional finite element model of a periodic unit cell of length $l$ with horizon $h=0.3 l$ : primary nodes (filled circles), slave nodes (hollow circles), virtual node with prescribed displacement (gray circle), and an expanded illustration of the considered interactions with different lengths $\xi$ (gray solid lines). Dashed arrows denote connections of slave nodes with primary nodes.

\section{NumeriCAL IMPLEMENTATION}

For numerical implementation, we use an analogy with the finite element analysis, adopted from [3], to represent a bond-based peridynamic system by a finite element truss model. Thus, individual bonds on the microscopic level are modelled by truss elements, so-called links, and the material parameters of the bonds, $c$ and $s_{0}$, are related to the macroscopic Young modulus $E$ and critical strain $\varepsilon_{0}$, respectively.

\subsection{ONE-DIMENSIONAL MODEL}

Damage localization and evolution of failure mechanisms in a one-dimensional periodic lattice submitted to positive strain is investigated for different forms of the critical strain length function. The geometry of an illustrative example of the finite element model of a one-dimensional periodic unit cell is depicted in Figure 3. All nodes in the periodic unit cell (except the last one) are modeled as primary nodes that carry independent degrees of freedom (horizontal displacements). To enforce periodicity, additional slave nodes are constructed as periodic images of primary nodes on one side of the periodic cell. The number of periodic slave nodes is given by the size of the horizon. Loading in the form of prescribed displacement is applied to the cell through one virtual node which is linked to all slave nodes simultaneously. The displacement of each slave node is evaluated as the sum of the unknown displacement of its master (one of the primary nodes) and the prescribed displacement of the virtual node.

Bonds, in the form of one-dimensional truss elements, are placed between all pairs of primary nodes as well as between primary nodes and slave nodes within the given horizon $h$; see the solid grey lines in Figure 3. The cross-sectional area of a truss element is the same for all links and it is scaled to provide a unit sum of overlapping link areas representing the macroscopic cross-sectional area. 
During the loading process, the evolution of damage in a macroscopic cell is modelled as a sequence of failures (breakages) of individual links followed by stress redistribution. The macroscopic damage $\omega$ can be evaluated for each point $x$ of the unit cell as

$$
\omega(x)=\frac{N_{\mathrm{f}}(x)}{N(x)}
$$

where $N$ is the number of all links passing through the given point $x$ and $N_{\mathrm{f}}$ is the number of broken links (i.e., those with $\mu=0$ ) passing through $x$.

Theoretically, it is possible that multiple links can reach the critical strain simultaneously. Then the subsequent solution is not unique and additional choices must be made to distinguish one link or a group of links preferred for failure in the given step. For an ideal lattice geometry without imperfections, such a situation occurs quite often, not only at the beginning of the loading, but also almost every time when broken links form a symmetric pattern. To avoid this phenomenon and to more closely mimic the behavior of real materials, the initial position of the primary nodes is randomized by a small perturbation with a uniform distribution and a maximum value $l \times 10^{-6}$. The macroscopic response of the randomized model is evaluated statistically based on multiple random realizations.

\subsection{Solution Algorithm}

To capture the exact sequence of breaking links, it is important to investigate not only the beginning of failure but also the full failure process of individual links and take into account that a new link can start failing before another (already failing) link becomes fully stress-free. For elastic-brittle links, failure means breaking, i.e., decreasing link stress under constant link strain. In this case, neglecting the possibility of partial unloading usually has only a minor effect on the macroscopic response. However, for elastic-damaging links with softening (which are not considered in this contribution), the possibility of partial unloading is essential.

It is obvious that the size of the loading increment to reach link failure or unloading is different in each step. Therefore, conventional solvers for an incremental nonlinear structural analysis are inconvenient for the present problem because they may miss the correct failure mechanism if the prescribed increment is too large. To solve the present problem exactly, an event-driven algorithm with step size control introduced in [4] has been employed. Since the bond-level constitutive law is piecewise linear, the step size can be selected such that the tangential stiffness matrix does not change during the step. Therefore, no equilibrium iteration is necessary. In one solution step, either a positive or a negative force increment is considered to achieve the onset of failure or complete failure of one link. Furthermore, an effective method of inelastic forces can be used to avoid the necessity of decomposing the tangential stiffness matrix in each step; for more details see section 4.2 in [4].

\section{RESUlts}

In this section, results are presented for the aforementioned finite element representation of the peridynamic model of a one-dimensional periodic cell with elastic-brittle interactions subjected to positive (average) strain. The modelled cell is considered to have a length of $l=1 \mathrm{~m}$, with the horizon $h=0.1 l$ and cross-sectional area $A=l^{2}$. The links have a constant Young modulus $E=100 \mathrm{~N} \mathrm{~m}^{-2}$ and critical strain function with a maximum value $\varepsilon_{0}=1 \times 10^{-2}$ and various forms, namely constant, linearly decreasing, and linearly increasing with link length.

\subsection{Convergence Study}

First of all, a convergence study of the peridynamic model with decreasing computational grid spacing is performed. For that purpose, five simulations with the same macroscopic parameters but with 10, 20, 50,100 , and 200 primary nodes have been performed. The simplest case of constant critical strain for different link lengths has been considered. The results are shown in Figure 4 in terms of normalized forcedisplacement diagrams, in which the elastic part of the displacement, $F / K_{\mathrm{el}}$, is subtracted from the total macroscopic displacement $u$ and only the inelastic part is plotted. $F$ is the macroscopic reaction force and $K_{\mathrm{el}}$ denotes the initial elastic stiffness. For the purpose of clarity, only one representative random realization is shown for each discretization.

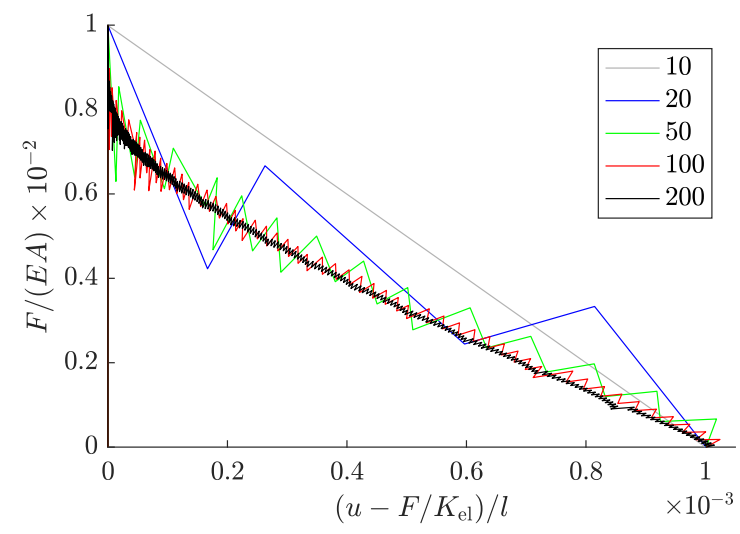

Figure 4. Convergence of normalized forcedisplacement diagrams (elastic part of displacements has been subtracted) for one-dimensional periodic lattice with fixed size $l$, horizon $h=0.1 l$, for discretizations with different numbers of nodes. For each discretization, only one representative random realization is shown.

The maximum force corresponding to the initial failure of the first link as well as the maximum displacement for the final damage mechanism are predicted exactly for all refinements. However, for a small number of nodes, significant oscillation can be 

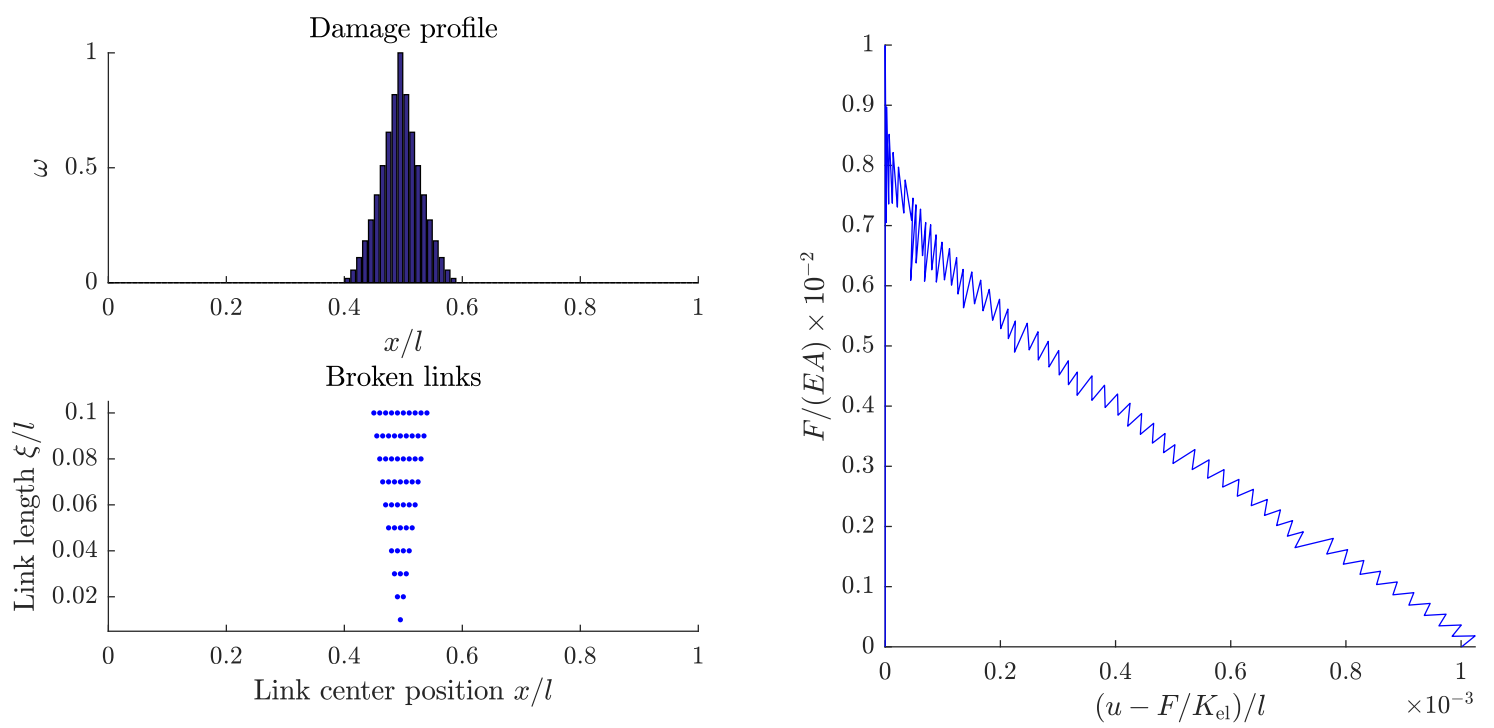

FIGURE 5. Results for constant critical strain: final damage profile (top left), positions and lengths of broken links (bottom left), and normalized force-displacement diagram where the elastic part of the displacement has been subtracted (right).
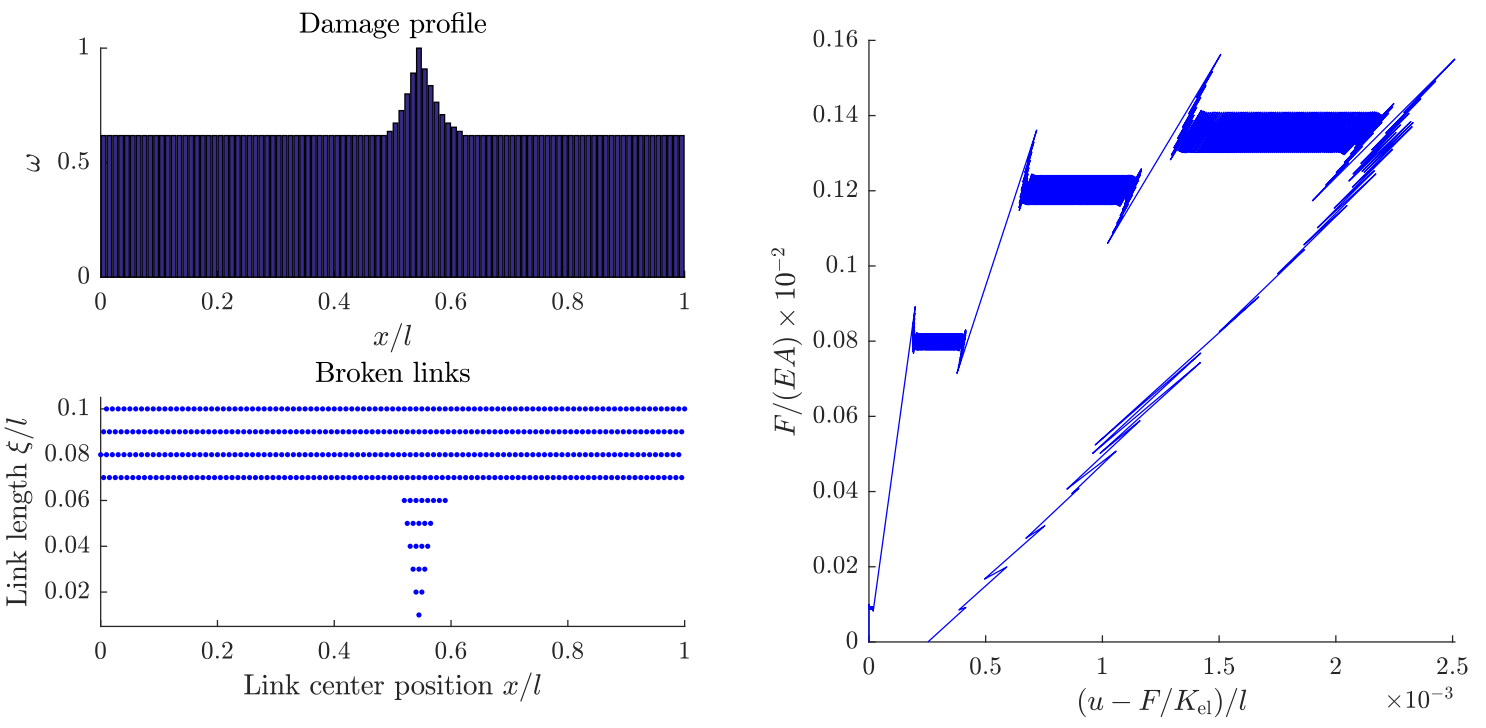

FIGURE 6. Results for critical strain linearly decreasing with link length: final damage profile (top left), positions and lengths of broken links (bottom left), and normalized force-displacement diagram where the elastic part of the displacement has been subtracted (right).

observed, while with an increasing number of nodes, the macroscopic response tends to a smooth curve. Discretization with 100 nodes turned out to be sufficient to capture the important characteristics of the macroscopic response.

Note that for 10 primary nodes, there is only one link between each pair of neighbouring nodes and therefore damage localizes in one step by failure of the first link and the corresponding response is a straight line, see the gray line in Figure 4.

\subsection{DAMAGE MECHANISMS}

In this subsection, the influence of the form of the critical strain function is analyzed. The discretization with 100 nodes is used based on the previous study. The results are shown in Figures 5, 6, and 7 for the constant, decreasing, and increasing functions of the critical strain, respectively. For each function, 10 independent random realizations have been evaluated to analyze the influence of randomness to the mechanisms of failure. However, for the purpose of clarity, only the results of one representative random realization are depicted.

The resulting mechanisms of failure are illustrated by the final damage profiles (top left in each figure), evaluated according to Eq. (8), and by the positions of all broken links (bottom left); here, each blue dot represent one broken link. The length of the broken link $\xi / l$ is plotted against the position of its center. The values are normalized by the cell size $l$. Moreover, the corresponding inelastic force-displacement diagram (right) is shown to illustrate the macroscopic 

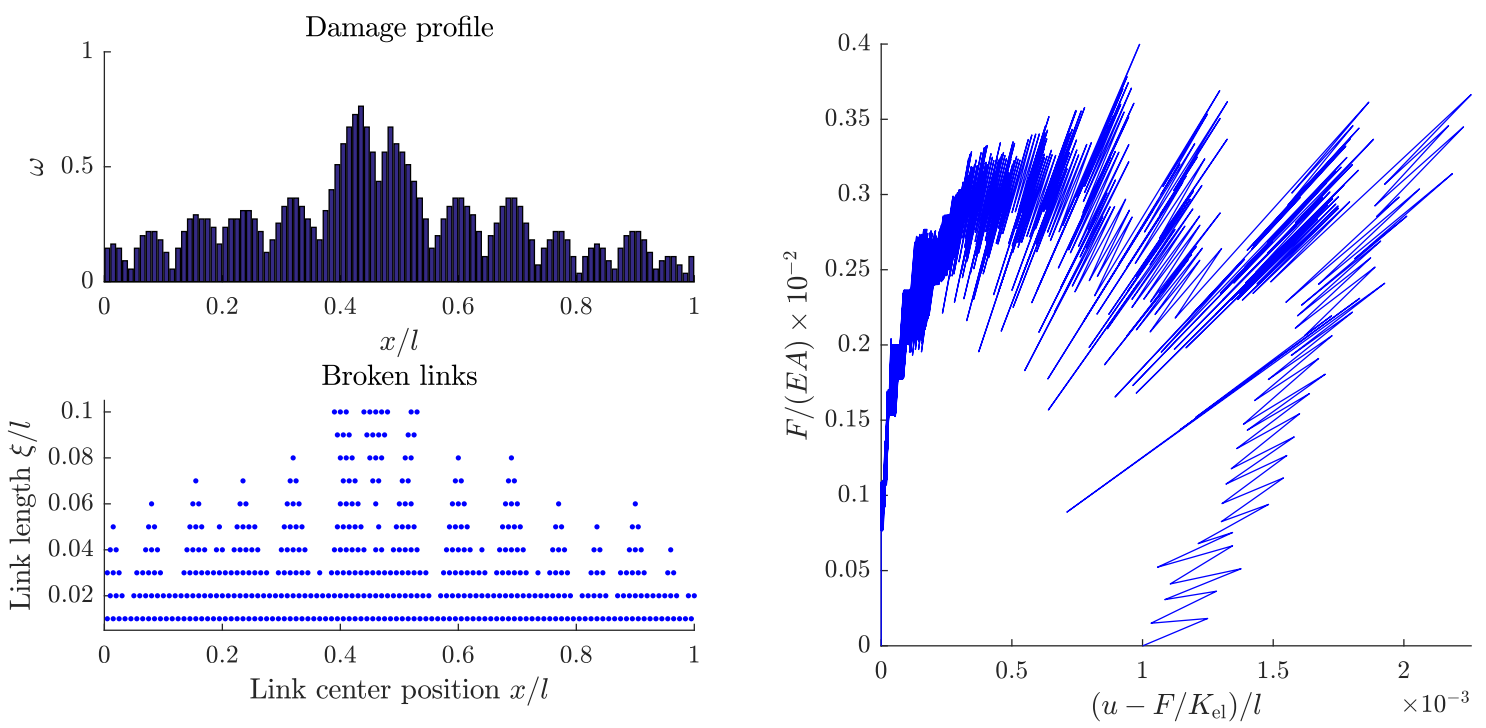

FigURE 7. Results for critical strain linearly increasing with link length: final damage profile (top left), positions and lengths of broken links (bottom left), and normalized force-displacement diagram where the elastic part of the displacement has been subtracted (right).

behavior during damage evolution.

\subsubsection{CONSTANT CRITICAL STRAIN}

In the initial (undamaged) configuration, the strain of the links is uniform. Consequently, for the first case of constant critical strain, there is no preferred length of links for failure in the first step. In our simulations, one of the shortest links is made weaker to initialize damage localization. Once the shortest link breaks, the stress is redistributed, two links in the second shortest layer spanning the broken link become the most loaded links and one of them fails. Then, each subsequent broken link is situated in the next longer layer spanning all previously broken links until the first link in the longest layer is reached. Once this sequence of propagating broken links from the shortest to the longest layer is finished, similar sequences follow starting from the second shortest to the longest, the third shortest to the longest, etc., until the final triangular pattern visible in Figure 5 (bottom left) is formed.

Note that if the shortest layer of links is not artificially favored at the beginning, the initial failure can occur for a different link length due to the randomization. However, in such a case, the closest shortest links break in the next step and the same failure sequences can be observed with only the exception of the first link.

For constant critical strain, the position of maximum damage $(\omega=1)$, i.e., the position of macroscopic failure, is dictated by the first broken link. The final damage mechanism is simple. The resulting failure pattern and associated failure properties such as fracture energy can be evaluated from the geometry of the cell. Also the force-displacement diagram does not exhibit any significant irregularities.

\subsubsection{DECREASING CRITICAL STRAIN}

In the case of a critical strain decreasing with link length, the longest layer of links is the weakest one and the first broken link appears in this layer. Subsequently, other links in the weakest layer are breaking one by one until the weakest layer is completely broken. This process is repeated for each next longest layer of links until a critical localization layer is reached. The gradual breaking of individual layers corresponds to horizontal parts of individual steps (jumps) in the force-displacement diagram. Particularly, four steps can be observed at the levels of normalized force about $0.01 \times 10^{-2}, 0.08 \times 10^{-2}$, $0.12 \times 10^{-2}$, and $0.135 \times 10^{-2}$, see Fig. 6 (right). From the macroscopic point of view, this process can be understood as a uniform growth of damage.

Once the critical localization layer is reached, broken links localize in a triangular pattern similar to the previous case of constant critical strain; see Fig. 6 (bottom left). In the force-displacement diagram, localization corresponds to the final unloading part, where both macroscopic force and macroscopic displacement are decreasing.

The position of maximum damage is not affected by the first broken link but it is determined during the localization of the damage in the critical localization layer.

\subsubsection{INCREASING CRITICAL STRAIN}

For the last case of increasing critical strain, the shortest layer of links is the weakest one and the first broken link appears here. However, in this case, damage of the first link is followed neither by damage of the whole weakest layer nor by localization. On the contrary, links continue breaking in different layers without a specific pattern. Consequently, the macroscopic damage is distributed randomly and no clear 
localization is observed. In the end, the majority of broken links are situated in several weakest layers. However, these weak layers (except the last one) are not completely broken. On the other hand, in several regions, damage significantly propagates also into layers of stronger links, see Fig. 7 (bottom left).

In the central region, the broken links form an unusual mechanism of failure where no macroscopic cross-section exhibits complete damage $(\omega=1)$, even when the macroscopic reaction force completely vanishes. Such a mechanism is formed by overlapping sliding parts. As a result of this, the final macroscopic strain is localized not only into one but into three segments between neighbouring nodes where two of them exhibit positive strain increments and the remaining one a negative strain increment of equal magnitude.

Unlike the previous cases of constant and decreasing forms of critical strain function, in this case, the order of broken links is unstable and the position and shape of the final damage pattern as well as the evolution of the damage before localization are affected by the randomization of the initial lattice geometry. As a result, significant oscillations can be observed in the force-displacement diagram, see Fig. 7 (right).

\section{Conclusions}

In this contribution, a finite element truss model representing a peridynamic system with elastic-brittle interactions has been introduced, together with an accurate and efficient event-driven numerical algorithm which is able to capture exactly not only the failure but also the full unloading of individual links. The convergence study of the presented model has shown that upon refinement the system tends to a smooth macroscopic response.

The influence of various forms of the critical strain function on the type of macroscopic response has been investigated for a one-dimensional periodic unit cell. Constant, decreasing, and increasing critical strain functions have been investigated and the resulting responses with different types of damage evolution have been analyzed, namely direct damage localization, uniform damage evolution preceding localization, and random damage distribution, respectively.

The presented results demonstrate that the concept of critical strain values depending on link length can be used to reach various types of macroscopic responses even for such a simple geometry (1D) and one of the simplest nonlinear material models (elasticbrittle). The significant advantage of this concept is that the presented elastic-brittle model can be fitted to represent various macroscopic behaviors, but at the same time the micro-level constitutive law remains piecewise linear and therefore the exact and efficient event-driven algorithm can be used.

In addition, the spectrum of different response types can be further extended by considering various micromodulus functions or by adopting elasticdamaging links with softening.

\section{LIST OF SYMBOLS}

$h$ Material horizon [m]

$\mathcal{H}_{x}$ Neighborhood of a particle

$V_{\mathbf{x}}$ Volume of a particle neighborhood

$\mathbf{x}$ Particle coordinates $[\mathrm{m}]$

u Displacement vector $[\mathrm{m}]$

$\ddot{\mathbf{u}}$ Acceleration vector $\left[\mathrm{m} \mathrm{s}^{-2}\right]$

$\rho$ Mass density $\left[\mathrm{kg} \mathrm{m}^{-3}\right]$

b Body force density $\left[\mathrm{N} \mathrm{m}^{-3}\right]$

$t$ Time [s]

$\boldsymbol{\xi}$ Relative position of two particles [m]

$\boldsymbol{\eta}$ Relative displacement of two particles [m]

f pairwise force function $\left[\mathrm{N} \mathrm{m}^{-6}\right]$

$f \quad$ scalar bond force $\left[\mathrm{N} \mathrm{m}^{-6}\right]$

$s$ Bond stretch $[-]$

$x$ Horizontal coordinate $[\mathrm{m}]$

$l \quad$ Cell size $[\mathrm{m}]$

$A$ Cross-sectional area of links $\left[\mathrm{m}^{2}\right]$

$\bar{u}_{x} \quad$ Prescribed horizontal displacement [m]

$u$ Total lattice displacement [m]

$F \quad$ Reaction force [N]

$K_{\mathrm{el}}$ Initial elastic stiffness $\left[\mathrm{N} \mathrm{m}^{-1}\right]$

$c$ Spring constant $\left[\mathrm{N} \mathrm{m}^{-2}\right]$

$s_{0} \quad$ Critical stretch for bond failure $[-]$

$E$ Young modulus $\left[\mathrm{N} \mathrm{m}^{-2}\right]$

$\varepsilon_{0} \quad$ Critical strain $[-]$

$\mu$ Damage function $[-]$

$\omega$ Damage [-]

$N$ Number of links [-]

$N_{f} \quad$ Number of broken links [-]

\section{ACKNOWLEDGEMENTS}

This research has been performed in the Center of Advanced Applied Sciences (CAAS), financially supported by the European Regional Development Fund (project No. CZ.02.1.01/0.0/0.0/16_019/0000778). Financial support received from the Czech Technical University in Prague under project SGS20/038/OHK1/1T/11 is gratefully acknowledged.

\section{REFERENCES}

[1] S. A. Silling. Reformulation of elasticity theory for discontinuities and long-range forces. Journal of the Mechanics and Physics of Solids 48(1):175-209, 2000.

[2] S. A. Silling, E. Askari. A meshfree method based on the peridynamic model of solid mechanics. Computers E structures 83(17-18):1526-1535, 2005.

[3] R. W. Macek, S. A. Silling. Peridynamics via finite element analysis. Finite Elements in Analysis and Design 43(15):1169-1178, 2007.

[4] M. Jirásek, Z. P. Bažant. Macroscopic fracture characteristics of random particle systems. International Journal of Fracture 69(3):201-228, 1994. 